\title{
Les flux du futur
}

\author{
M. Davidts \\ Co SOCACHIM, 149 Avenue du Capricorne, 1200 Bruxelles, Belgique
}

\begin{abstract}
Résumé :
$L$ 'analyse par fluorescence de rayons $X$ est une méthode très fiable dont les résultats dépendent très largement des techniques de préparation de l'échantillon.

La fusion à l'aide de borates est reconnue comme la technique actuelle la plus fiable.Les propriétés physico-chimiques du fondant sélectionné joue un rôle prépondérant. Les caractéristiques des nouveaux fondants fondus anhydres trés faiblement hygroscopiques une ayant une perte au feu minimale, une parfaite homogénéité,une granulométrie controllée,une densité élevée améliorent considérablement la fiabilité et la reproductivité des analyses.Des mélanges de fondants spécifiques homogène et stables sont disponibles
\end{abstract}

\begin{abstract}
:
The analysis by $\mathbf{X}$ ray fluorescence is a reliable technique but very dependant on the sample preparation method.Fusion with borate is recognised as the most accurate technique,the selection and the quality of the selected flux plays a major role.Thanks to a original process of fabrication of prefused anhydrous fluxes, the required criteria are now currently available : various purity grades,no loss of ignition, perfect homogeneity, low specific surface,controlled granulometry, heavy density. Homogeneous prefused mixes for particular applications are available with the same degree of reliability.
\end{abstract}

\subsection{PREPARATION DE L'ECHANTILLON}

L'analyse par fluorescence de rayons-X n'est pas une méthode absolue. Elle est basée essentiellement sur des comparaisons avec des standards. C'est pourquoi la précision et la fiabilité de la méthode dépendent en très grande partie du soin apporté à la préparation de l'échantillon.

A partir de l'échantillon de base apporté au laboratoire, il convient de préparer une entité ayant en tout point exactement la même composition physique et chimique. Cette entité est appelée échantillon final ou spécimen.

\subsection{TECHNIQUES DE PREPARATION DU SPECIMEN.}

Deux techniques sont actuellement très largement répandues pour les préparations d'échantillons pulvérulents, à savoir la confection de pastilles ou tablettes comprimées et la fabrication de perles par fusion.

- La technique de pastillage consiste à partir de l'échantillon livré au laboratoire d'opérer un broyage le plus fin et le plus homogène que possible.Le but est d'éliminer du mieux possible la structure cristalline et la distribution des particules. La poudre ainsi obtenue est pressée avec éventuellement l'aide d'adjuvants de broyage ou de cohésion.

- La fusion consiste en la dissolution de l'échantillon dont les éléments ont été préalablement oxydés par un solvant porté à haute température $\left(1050-1100^{\circ} \mathrm{C}\right)$ dans un creuset en alliage platine or. Le solvant utilisé s'appelle fondant ou flux de fusion.En général on utilise des borates, métaborates. Le 
produit de fusion fondu est coulé dans une coupelle à fond plat.Après refroidissement on obtient une pastille de verre dénommé perle de fusion de structure parfaitement homogène et vitreuse.

Les critères de sélection de l'une ou l'autre méthode dépend du degré de précision et fiabilité demandée en fonction de la vitesse d'obtention des résultats et du coût.

Ces deux méthodes seront brièvement décrites avec leurs avantages et inconvénients.

\subsection{LIMITATION de l'analyse par Fluorescence de rayons-X.}

Seule une petite portion du spécimen préparé sera "vue" et analysé par le spectromètre.

A cause du au faible pouvoir de pénétration des rayons $\mathrm{x}$ seule les premières couches de particules sont en général analysées. Il est donc primordial que l'ensemble de ces couches soient aussi homogène et représentative que possible.

L'analyste et les responsables du laboratoire se doivent de bien évaluer les avantages et inconvénients des deux méthodes. S'ils optent pour la solution de confection de pastilles compressées, ils doivent considérer tous les effets possibles d'hétérogénéité et les limitations de la précision et de la fiabilité. Les effets ayant une influence négative sur la fiabilité et la précision sont décrits ci-dessous.

- Effet de grain ou de particule

Sur des gros grains les rayons $\mathbf{x}$ donnent des informations sur la composition spécifique des éléments contenus dans ce grain, tandis que sur de petites particules les informations reçues seront représentatives de la composition générale.

\section{- Rugosité et état de la Surface}

Lorsque des grains sont volumineux les rayons $\mathrm{X}$ émis sont partiellement réabsorbés, pouvant dans certains cas être complètement absorbés : effet opacifiant.

La sensibilite du spectromètre varie en fonction de la distance entre le tube à rayons $\mathrm{x}$ et l'échantillon.La planéité de la préparation devra être donc aussi parfaite que possible.

\section{- Distribution granulométrique}

L'intensité des rayons $\mathbf{x}$ émis varie de façon significative en fonction de la distribution granulométrique malgré tout le soin apporté au broyage on ne peut éviter ce phénomène.

Il est extrêmement difficile, pour ne pas dire impossible d'obtenir des standards moulus ayant la même composition granulométrique que celle de l'échantillon à analyser.

\section{- Effet de matrice}

Les corrections mathématiques sont rendues extrêmement difficiles, voire impossibles par suite de la difficulté de décrire avec précision un modèle d'une structure de poudre broyée.

\section{- Détermination de la composition finale}

Pour les mêmes raisons qu'évoquées ci-dessus, les calculs basés sur l'analyse par régression ne sont applicables que pour des particules de même composition, de même taille et de forme géométrique identique.

\section{- Orientations préférentielles}

Lorsqu'une poudre est comprimée, les particules non sphériques, comme par exemple à bord plat et allongées ont une tendance plus grande à se mettre parallèlement contre la surface, cela se traduit par des irrégularités de composition vue par le spectromètre.

\section{- Ségrégation}

Ce phénomène est difficilement évitable durant les différentes techniques d'homogénéisation de l'échantillon. On peut cependant minimiser cet effet en ajoutant des liants : mais il faut bien contrôler que cet ajout n'amène des hétérogénéités supplémentaires, ce qui n'est pas toujours respecté strictement. 


\section{- Liaisons chimiques}

Il est aussi malaisé de mesurer les effets des réactions inter éléments : attraction, répulsion, formation de nouvelles entités, etc...

On essaye de minimiser ces sources d'hétérogénéité en broyant très finement et longuement dans des broyeurs en agate par exemple. Mais cela aux dépens de la rapidité de cette technique qui en est d'ailleurs le plus grand avantage.

Pour obtenir des résultats passablement fiables, le temps mis par cette méthode est identique à celui de la fusion.

Dans le cas d'option pour des systèmes automatisés, il faut envisager l'effet de pollution inter échantillons et l'accroissement de temps nécessaire à la décontamination.

\subsection{AVANTAGES DE LA FUSION}

La fusion a pour objet d'éliminer toutes les effets dus aux hétérogénéités décrites ci-dessus et en particulier l'élimination de l'effet de matrice.

On solubilise à $1000-1100^{\circ} \mathrm{C}$ les éléments sous forme d'oxydes, dans un fondant ou un mélange de fondants généralement à base de tétraborate et/ou métaborate de lithium.Le phénomène de dissolution des oxydes dans les borates est bien connu des chimistes sous le nom de la méthode de la " perle de borax". Le produit de fusion est ensuite coulé dans une coupelle en métaux précieux et l'on force le refroidissement afin d'éviter la cristallisation.

Le résultat de cette opération permet l'obtention d'un disque de structure vitreuse et homogène possédant une surface parfaitement plane.L'utilisation de coupelles de coulée en métal ODS,platine-or à grain stabilisé nettement moins déformables que le matériel classique en platine-or est fortement recommandé.(2).

Cette méthode est applicable à la grande majorité des éléments dont le nombre atomique est supérieur à 10 .

Le traitement chimique est aisé : addition d'oxydants, de standards interne, d'absorbant, d'halogénures etc.

La rapidité et la simplicité de procédure pour la préparation de perles d'échantillons et de standards certifiés, ainsi que la possibilité de conserver les échantillons durant de longues périodes sont tels que cette technique est de plus en plus utilisée et en pleine expansion pour les analyses de contrôle et de routine.

Les résultats d'analyse obtenus sont très fiables et reproductibles.

\subsection{INCONVENIENTS DE LA FUSION}

Elle n'est pas applicable qu'aux éléments oxydés dont le poids atomique est supérieur à 10 ainsi qu'au carbone et au mercure formant des oxydes volatils et aux métaux précieux, $\mathrm{Pt}, \mathrm{Au}, \mathrm{Ag}$, difficilement oxydables.

La dilution des éléments mineurs et légers résulte en une perte de sensibilité.L'emploi de flux de haute pureté permet de retrouver cette perte.

Il faut tenir compte de la Perte au feu ou du Gain au feu.

Elle est plus lente que le pastillage à cause du temps de fusion ( 5 à 8 minutes). L'utilisation de certaines machine de fusion permettent la préparation simultanée de 2 à 12 échantillons.Le coût par fusion serait sensiblement supérieur, en moyenne de 4,5 FF/fusion pour le matériel en platine et le fondant.( On ne tient pas compte du temps mis à nettoyer convenablement le matériel de broyage et des presses pour éviter les contaminations inter échantillons) 
Quelles devraient être les caractéristiques d'un bon flux ? La réponse serait: " transparent comme l'eau". Malheureusement un bon nombre d'éléments polluent les fondants, citons les plus courants comme : $\mathrm{Fe}, \mathrm{Si}, \mathrm{Al}, \mathrm{Ca}, \mathrm{S}, \mathrm{K}, \mathrm{Mg}, \mathrm{Na}$.

\subsection{PURETE ANALYTIQUE :}

La pureté analytique, en ce qui concerne le choix du fondant le plus approprié pour une analyse donnée, est heureusement un concept relatif.

L'analyste doit avoir la possibilité de sélectionner le fondant le plus approprié à ses besoins. ICPH lui offre cet avantage en procurant un certificat d'analyses des lots de fabrication. Une spécification d'achat, établie par le laboratoire, est fortement conseillée.

Le critère de choix analytique est basé sur la formule suivante :

$\mathrm{Ca}=\mathrm{Cf} \times$ rapport fondant/échantillon

où $\mathrm{Ca}=$ concentration apparente, $\mathrm{Cf}=$ concentration de l'élément dans le fondant.

Par exemple la concentration apparente pour un fondant contenant $20 \mathrm{ppm}$ d'Al pour une dilution de 7 sera de : $0,0020 \% \times 7 / 1=0,014 \%$.

Si l'on désire une précision de $1 \%$, la méthode n'est applicable pour autant que l'échantillon à analyser ne contienne qu'au maximum $1,4 \% \mathrm{~d}$ 'Al.

On peut donc résoudre ce problème par la SELECTION de LOT de FABRICATION.

Etant donné la plus grande sensibilité des spectromètres modernes, les fondants classiques de pureté $3 \mathrm{~N}$ sont souvent dépassés.Pour un nombre croissant d'applications en géologie, minéralogie et analyse de métaux, etc. des fondants de HAUTE PURETE sont de plus en plus demandés et utilisés.

Des fondants de pureté $4 \mathrm{~N}$ adaptés aux critères actuels sont actuellement disponibles sur le marché. (1) Ces qualités sont livrées avec certificat d'analyse du lot de production.

\subsection{HOMOGENEITE :}

Sachant que la fluorescence de rayons $X$ est une méthode d'analyse relative, basée sur des comparaisons, il est important que d'une analyse à l'autre l'on puisse reproduire exactement les mêmes conditions.

Dés lors l'utilisation de fondants homogènes est primordiale pour l'obtention de valeurs fiables.

L'homogénéité des lots de fondants est donc primordiale. Pour obtenir les meilleurs résultats possibles, un fabriquant n'hésite pas à homogénéiser de grandes quantités de matières premières.

Diverses homogénéisations ont lieu aux différents stades de fabrication.

Les fondants fondus anhydres et le mélange fondu de divers composants présentent une meilleure homogénéité que les fondants classiques du marché sous forme de poudres de granulométrie variable et hygroscopique. En effet chaque particule du fondant fondu a exactement la même composition. Les caractéristiques physico-chimiques décrites ci-après sont également des éléments garantissant une meilleure homogénéité.

\subsection{PROPRIETES PHYSIQUES}

Afin d'éviter les erreurs éventuelles de pesage par suite de poussières, les fondants sont tamisés dans le but d' obtenir une granulométrie bien définie. En général des tailles de particules comprises entre 100 et 
500 microns sont idéales. Une conséquence directe est la meilleure fluidité de ce type de fondant .Cette propriété est mise à profit dans les systèmes de pesée automatique.

La densité des fondants fondus anhydres est d'environ de $1 \grave{a} 1,1$, ce qui permet une meilleure homogénéisation entre l'échantillon et le flux .Les pertes possibles lorsqu'on remplit des creusets de faible volume sont ainsi évitées.

Un traitement spécial permet de supprimer les tensions internes des particules de fondant évitant les projections hors du creuset durant la montée en température des creusets.

Les fondants fondus anhydres ne sont plus la cause de moussage et formation de bulles.

La faible surface spécifique des particules ainsi que leurs surfaces dures et vitreuses retardent considérablement les phénomènes de réhydratation et recarbonatation et permet le stockage prolongé dans les silos des doseurs automatiques.

\subsection{PERTE AU FEU :}

Les fondants anhydres ont une perte au feu après fabrication de $0,03 \%$, qui n'excède pas $0,1 \%$ après un an de stockage. Ces valeurs sont en deçà des erreurs d'analyses admises.Il n'est donc plus nécessaire de tenir compte de la perte au feu dans les calculs de corrections.

De nombreux flux actuellement en vente montrent cepandant des valeurs de pertes au feu variables pouvant aller de $1,5 \%$ jusqu'à $4 \%$.

\subsection{REPRODUCTIBILITE}

Il est pratiquement impossible de fabriquer deux lots de fondants identiques possédant la même composition analytique en éléments indésirables.C'est pourquoi nous suggérons aux utilisateurs de couvrir leur besoin par une commande d'une plus grande quantité de fondant en provenance d'un seul et même lot de fabrication.

Afin de réduire les écarts entre 2 lots différents, les matiéres premières sont selectionnées en fonction des critères de pureté exigée.Par l'homogénéisation de grande quantité de matière première., le carbonate de lithium en particulier,on améliore considérablement le reproductiblité entre les différents lots de fabrication.

\subsection{MELANGES SPECIAUX}

Différents mélanges sont proposés à l'utilisateur (1):

- Mélanges fondus anhydres : borates, métaborates, oxydes de lanthane, strontium, cobalt, fluorure de lithium etc.

- Mélanges physiques homogènes : de produits et fondants fondus avec ajout d'oxydants,nitrates, halogénures etc. METHODES DE PREPARATION

\subsection{FONDANTS DISPONIBLES}

Un avantage non négligeable de l'utilisation des fondants est la variété de choix, permettant de choisir la meilleure composition du ou des fondants pour une application déterminée.

Toutes les combinaisons en proportion variable sont possibles à partir de produits comme:

- tétraborate de lithium

- métaborate de lithium

- fluorure de lithium 
- oxyde de lanthane

- tétraborate de lithium

- anhydride borique

- oxydes divers, oxyde de cobalt,vanadium,strontium.

- polyphosphates, métaphosphates etc.

\subsection{SELECTION DES FONDANTS}

Le tétraborate de lithium est considéré comme un flux de caractère acide et dissout les oxydes basiques comme : $\mathrm{Na2O}, \mathrm{CaO}, \mathrm{MgO}, \mathrm{K} 2 \mathrm{O}$, Silicates, carbonates etc.

Le métaborate de lithium est un fondant "basique" permettant une meilleure dissolution des oxydes acides comme: SiO2, phosphates, $\mathrm{Al} 2 \mathrm{O} 3$, sulfates.

Les oxydes métalliques et amphotères sont plus facilement solubilisés par un mélange de tétraborate et métaborate de lithium.

Certaines écoles préconisent le métaborate comme fondant de base avec addition de 20

à $36 \%$ de tétraborate de lithium

Le tétraborate de lithium est actuellement le flux le plus couramment utilisé, il est aussi le moins onéreux. Il permet l'obtention de perles stables. Son champ d'application est vaste :

analyses des aciers, métaux, des ferro-alliages, en géologie, clincker, etc.

Le métaborate est mélangé avec le tétraborate pour augmenter la palette de solubilité et la vitesse de dissolution, en particulier pour l'alumine. Il diminue la température de fusion et permet, sous certaines conditions de capter des volatils comme les sulfures. Les perles obtenues avec le métaborate pur sont moins stables que les perles de tétraborate de lithium.

Les mélanges méta/tétraborate permettent l'obtention de perles fort stables et évitent des phénomènes de cristallisation durant le refroidissement de la perle.

Le tétraborate de sodium est de moins en moins utilisé.Le sodium est souvent inclu dans le programme analytique.De plus le sodium est associé avec les alcalins comme le $\mathrm{K}, \mathrm{Mg} \mathrm{Ca}$ ainsi que le $\mathrm{S}$.

Néanmoins il reste un excellent solvant des oxydes métalliques tel que le $\mathrm{Cr}, \mathrm{Ni}, \mathrm{Fe}$.

Le mélange lithium/sodium tétraborate $50: 50$ est encore utilisé,mais tend à disparaitre.

Les absorbants comme le lanthane sont également de moins en moins utilisés au vu de la disponibilité plus grandes des programmes de calculs de corrections intégrés sur le spectromètre.En outre l'utilisateur désire également étendre ses analyses à la gamme des éléments dont le rayonnement était auparavant absorbé par ces agents dits "absorbants" ou opacifiants.

\subsection{TECHNIQUE DE PREPARATION DES PERLES}

La première étape consiste à oxyder les éléments à leur plus haut degré d'oxydation, quand cela s'avère nécessaire ( produits métallurgiques, aciers etc.)

On ajoute, en général des oxydants dont le plus efficace serait le nitrate de lithium, mais des mélanges d'oxydants comme le nitrate de strontium, potassium, sodium permet une oxydation étagée à partir de $400^{\circ} \mathrm{C}$ jusqu'à $800^{\circ} \mathrm{C}$. Le carbonate de lithium est souvent recommandé dans les cas difficiles d'oxydation de ferro-alliages.(Fe-Cr).

Afin d'éviter la destruction des creusets en platine par les métaux non oxydés, il convient de tapisser le fond du creuset avec du fondant et d'ajouter l'échantillon intimement mélangé avec les oxydants. On recommande d'élever la température progressivement de 400 à $800^{\circ} \mathrm{C}$ durant 2 à 3 minutes, soit dans un four à moufle ou grâce au programme d'oxydation prévu sur les perleuses (par exemple : la Perl'X3) ou d'autres fluxeurs possédant cette possibilité et de procéder ensuite à la fusion à $1100-1200^{\circ} \mathrm{C}$. L'ajout de quelques milligrammes d'halogénures du type LiF, iodures, iodates permet d'améliorer l'écoulement de la matière en fusion hors du creuset et d'éviter le collage et l'effet de goutte rémanente. Dans le tableau 1 , nous avons reportés quelques méthodes de fabrication de perles.

On peut analyser les éléments volatils comme les sulfures en les transformant en sulfates. On choisit un mélange fondant à température de fusion relativement basse comme le FLUORE - X 65 par exemple.On 
melange intimement une quantité d'oxydants, en général du nitrate de lithium ou mélange d'oxydant avec l'échantillon en proportion de 4 à 1 .Afin d'éviter l'attaque du matériel platine, on tapisse le fond du creuset par une couche de fondant et en y ajoutant l'échantillon mélangé avec l'oxydant sur lequel on aura rajouté le reste de fondant. On chauffe progressivement de 400 à $800^{\circ} \mathrm{C}$ durant $2-3$ minutes, ensuite on monte la température de fusion jusqu'à $1000^{\circ} \mathrm{C}$ maximum jusqu'à ce que le mélange soit entièrement fusionné.On poursuit la fusion comme à l'ordinaire. (Méthode mise au point par Claisse).

Tableau 1

\begin{tabular}{|c|c|c|c|c|c|c|}
\hline FRX & Perle & $\begin{array}{l}\text { Méthode } \\
\text { générale }\end{array}$ & \begin{tabular}{|l|} 
Poussière de \\
Gaz
\end{tabular} & Scories & $\begin{array}{l}\text { Fe-Cr-C } \\
(1)\end{array}$ & $\begin{array}{l}\text { Fe-Si } \\
(2)\end{array}$ \\
\hline Echantillon & $\begin{array}{l}\text { Quantité } \\
\text { Granulo. }\end{array}$ & $\begin{array}{l}0,300 \mathrm{~g} \\
<80 \mu\end{array}$ & $\begin{array}{l}0,250 \mathrm{~g} \\
<80 \mu \\
\end{array}$ & $\begin{array}{l}0,500 \mathrm{~g} \\
<100 \mu\end{array}$ & $\begin{array}{l}0,250 \mathrm{~g} \\
<200 \mu \\
\end{array}$ & $\begin{array}{l}0,250 \mathrm{~g} \\
<200 \mu \\
\end{array}$ \\
\hline Fondant & $\begin{array}{l}\text { Type } \\
\text { Origine } \\
\text { Quantité }\end{array}$ & $\begin{array}{l}\text { Fluore-X } 65 \\
\text { I.C.P.H. } \\
5,000 \mathrm{~g} \\
\end{array}$ & $\begin{array}{l}\text { ATL100 } \\
\text { I.C.P.H. } \\
5,000 \mathrm{~g} \\
\end{array}$ & $\begin{array}{l}\text { ATL100 } \\
\text { I.C.P.H. } \\
5,500 \mathrm{~g} \\
\end{array}$ & $\begin{array}{l}\text { ATL100 } \\
\text { I.C.P.H. } \\
5,500 \mathrm{~g} \\
\end{array}$ & \begin{tabular}{|l} 
ATL100 \\
I.C.P.H. \\
5,000 \\
\end{tabular} \\
\hline Additifs & Halogén. & LiI : $0,020 \mathrm{~g}$ & & LiI: 0,02 & & LiI : 0,040 \\
\hline \multirow[t]{7}{*}{ Oxydant } & \multirow{7}{*}{\begin{tabular}{|l|}
$\mathrm{LiNO3}$ \\
$\mathrm{NaNO} 3$ \\
$\mathrm{KNO} 3$ \\
$\mathrm{Sr}(\mathrm{NO} 3) 2$ \\
$\mathrm{Li} 2 \mathrm{CO} 3$ \\
$\mathrm{Na} 2 \mathrm{CO} 3$ \\
$\mathrm{NaIO} 4$ \\
\end{tabular}} & $0,200 \mathrm{~g}$ & & & $0,750 \mathrm{~g}^{*}$ & $0,750 \mathrm{~g}^{*}$ \\
\hline & & & & & $*$ ou & * ou \\
\hline & & & $0,500 \mathrm{~g}$ & & $0,125 \mathrm{~g}$ & $0,125 \mathrm{~g}$ \\
\hline & & & & $0,500 \mathrm{~g}$ & $0,250 \mathrm{~g}$ & $0,250 \mathrm{~g}$ \\
\hline & & & & & $2,000 \mathrm{~g}$ & \\
\hline & & & & & & \\
\hline & & & & & $0,020 \mathrm{~g}$ & \\
\hline Pre-Oxyd. & $\begin{array}{l}\text { Temp. } \\
\text { Durée }\end{array}$ & $\begin{array}{l}900-950^{\circ} \mathrm{C} \\
5-6 \text { min. }\end{array}$ & $\begin{array}{l}900^{\circ} \mathrm{C} \\
5-6 \mathrm{~min} .\end{array}$ & $\begin{array}{l}900{ }^{\circ} \mathrm{C} \\
5-6 \mathrm{~min} .\end{array}$ & $\begin{array}{l}1200^{\circ} \mathrm{C} \\
6 \mathrm{~min} .\end{array}$ & $\begin{array}{l}900^{\circ} \mathrm{C} \\
6 \mathrm{~min} .\end{array}$ \\
\hline Fusion & $\begin{array}{l}\text { Temp. } \\
\text { Durée }\end{array}$ & $\begin{array}{l}1.100^{\circ} \mathrm{C} \\
4 \mathrm{~min} .\end{array}$ & $\begin{array}{l}1250^{\circ} \mathrm{C} \\
4 \mathrm{~min}\end{array}$ & $\begin{array}{l}1100^{\circ} \mathrm{C} \\
3,25 \mathrm{mi} .\end{array}$ & $\begin{array}{l}1200^{\circ} \mathrm{C} \\
3 \mathrm{~min}\end{array}$ & $\begin{array}{l}1200^{\circ} \mathrm{C} \\
3,5 \mathrm{~min}\end{array}$ \\
\hline $\begin{array}{l}\text { Creuset } \\
\text { Coupelle } \\
\end{array}$ & $\begin{array}{l}\text { Qualité } \\
\text { Qualité }\end{array}$ & $\begin{array}{l}\mathrm{Pt} / \mathrm{Au} 95: 5 \\
\mathrm{Pt} / \mathrm{Au} 95: 5\end{array}$ & $\begin{array}{l}\mathrm{Pt} / \mathrm{Au} \\
\mathrm{Pt} / \mathrm{Au}\end{array}$ & & $\begin{array}{l}\text { ODS (3) } \\
\text { ODS } \\
\end{array}$ & $\begin{array}{l}\text { ODS } \\
\text { ODS }\end{array}$ \\
\hline \multicolumn{7}{|c|}{$\begin{array}{l}\text { Pré-oxydation : les meilleurs résultats sont obtenus dans creusets } \mathrm{Pt} / \mathrm{Au} \text { 95:5 dans un four } \\
\text { à moufle porté de } 400^{\circ} \mathrm{C} \text { à } 800^{\circ} \mathrm{C} \text {. } \\
\text { (1) méthode applicable pour les ferro-alliage carburés. } \\
\text { (2) méthode applicable pour les alliages de type : Fe-B, Fe-Ti-Al, Fe-Cr, Fe-Mn, Fe-Si, } \\
\text { Fe-Si-Mn, Fe-Nb. } \\
\text { 3) ODS matériel platine à grain stabilisé provenant de la firme Engelhard-CLAL. }\end{array}$} \\
\hline
\end{tabular}

Nous recommandons l'utilisation de quelques mélanges d'oxydants prêts à l'emploi ;dont ceux rassemblés dans le tableau 2 : 
lithium carbonate : $\quad 40 \%$

lithium nitrate : $\quad 15 \%$

strontium nitrate : $\quad 5 \%$

potassium nitrate: $\quad 5 \%$

sodium nitrate : $\quad 5 \%$

lithium tétraborate : $30 \%$ lithium nitrate : $\quad 60 \%$

strontium nitrate $\quad 20 \%$

potassium nitrate $20 \%$

lithium tétraborate $90 \%$

lithium carbonate $10 \%$

Tableau 2

\subsection{METHODE DE PREPARATION DES FONDANTS}

Le tableau 3 compare les avantages des fondants du futur ( Fondants fusionnés anhydres, de densité élevée) avec les fondants traditionnels.Les caractéristiques physico-chimiques des produits de réactions finals sont déterminés par la méthode de préparation.

\begin{tabular}{|c|c|c|c|c|}
\hline & VOIE HUMIDE & VOIE SECHE & $\begin{array}{l}\text { FUSION } \\
\text { DIRECTE }\end{array}$ & F.A.F. (1) \\
\hline PROCEDE & $\begin{array}{l}\text { Suspension aqueuse } \\
\text { suivit d'un séchage à } \\
450^{\circ} \mathrm{C} \text {. } \\
\text { homogénéisation }\end{array}$ & $\begin{array}{l}\text { Mélange de poudre } \\
\text { suivie d'une } \\
\text { calcination } \\
\text { A } 600^{\circ} \mathrm{C} \text {. } \\
\text { homogénéisation }\end{array}$ & $\begin{array}{l}\text { Mélange de poudre } \\
\text { suivie de la fusion. } \\
\text { homogénésation }\end{array}$ & $\begin{array}{l}\text { Mélange de poudre } \\
\text { suivie d'une } \\
\text { calcination. } \\
\text { Fusion ì } 1250^{\circ} \mathrm{C} \\
\text { Broyage, recuì à } \\
600^{\circ} \mathrm{C} \text {, tamisage, } \\
\text { homogénéisation }\end{array}$ \\
\hline $\begin{array}{l}\text { température de } \\
\text { réaction }\end{array}$ & $450^{\circ} \mathrm{C}$ & $600^{\circ} \mathrm{C}$ & $1250^{\circ} \mathrm{C}$ & $1250^{\circ} \mathrm{C}$ \\
\hline $\begin{array}{l}\text { Nature des produits } \\
\text { de réaction. }\end{array}$ & $\begin{array}{l}\text { Poudre légère à haute } \\
\text { surface spécifique. }\end{array}$ & $\begin{array}{l}\text { Poudre légère à haute } \\
\text { surface spécifique. }\end{array}$ & $\begin{array}{l}\text { Verre de haute densité } \\
\text { à faible surface } \\
\text { spécifique. }\end{array}$ & $\begin{array}{l}\text { Verre de haute densité } \\
\text { à faible surface } \\
\text { spécifique. }\end{array}$ \\
\hline Qualité & Poudre hétérogène & $\begin{array}{l}\text { Granulats } \\
\text { hétérogènes. }\end{array}$ & $\begin{array}{l}\text { Polyèdres non } \\
\text { homogènes avec } \\
\text { inclusion d'H2O et de } \\
\mathrm{CO} 2 \text {. } \\
\end{array}$ & $\begin{array}{l}\text { Polyèdres } \\
\text { homogènes anhydres } \\
\text { sans inclusions. }\end{array}$ \\
\hline teneur en $\mathrm{HO}$ & ca $3 \%$ & ca 1,5 à $2 \%$ & $\begin{array}{l}\text { ca } 0,5 \% \text { mais très } \\
\text { irrégulier. }\end{array}$ & $0,003 \%$ typique \\
\hline Hygroscopicité & Haute & Haute & aucune & aucune \\
\hline Densité apparente & 0,3 & 0,5 & 1,0 & 1,0 \\
\hline Structure & Spongieuse.. & Spongieuse. & $\begin{array}{l}\text { particules pleines } \\
\text { avec inclusion de } \\
\text { bulles. }\end{array}$ & particules pleines. \\
\hline Formule Chimique & $\begin{array}{l}\mathrm{Li} 2 \mathrm{~B} 4 \mathrm{O} 7, \mathrm{n} \mathrm{H} 2 \mathrm{O} \\
\mathrm{n}=0,2 \mathrm{H} 2 \mathrm{O}\end{array}$ & $\begin{array}{l}\mathrm{Li} 2 \mathrm{~B} 4 \mathrm{O} 7, \mathrm{~m} \mathrm{H} 2 \mathrm{O} \\
\mathrm{n}=0,1 \mathrm{H} 2 \mathrm{O}\end{array}$ & $\begin{array}{l}\mathrm{Li} 2 \mathrm{~B} 4 \mathrm{O} 7+ \\
? \mathrm{H} 2 \mathrm{O}, ? \mathrm{CO} 2\end{array}$ & \begin{tabular}{|l} 
Li2B4O7 \\
ANHYDRE
\end{tabular} \\
\hline
\end{tabular}

Le procédé de fabrication choisi est déterminant pour les propriétés finales du fondants Dans le procédé de la voie humide les matiéres premières sont mises en suspension dans l'eau, aprés réaction à $450^{\circ} \mathrm{C}$, on élimine l'eau par séchage.Le produit obtenu est une fine poudre pulvérulente de densité faible et très hygroscopique du fait de sa haute surface spécifique.La teneur en eau de l'ordre de $3 \%$ est fort élevée. 
Dans le procédé dit de voie séche,comme le sont la plupart des fondants classiques,le fondant est obtenu par mélange des matières premiéres suivit d'une calcination à $600^{\circ} \mathrm{C}$.Les produits de réactions se présentent-sous la forme de granulats et de poudre de faible densité,de structure spongieuse à haute surface spécifique et donc très hygroscopique.La teneur en eau de l'ordre de 1,5 à $2 \%$ est élevée et il faudra done en tenir compte lors des calculs de perte au feu.

Les fondants anhydres fondus (F.A.F.) (1) sont obtenus au départ de le même façon que par voie séche.Aprés homogénéisation le produit est fusionné à $1250^{\circ} \mathrm{C}$ dans un four en alliage platine or.Aprés un recuit afin d'éliminer les tensions le produit vitreux obtenu est broyé et tamisé.La teneur en eau des fondants obtenus par cette méthode sont de l'ordre de 0,003 à $0,01 \%$.L'aspect physique est une poudre d'aspect cristalline, de densité élevée et à faible surface spécifique.

La méthode dite de fusion directe consiste à fusionner directement le mélange de matières premières.On ne peut éviter l'emprisonement de bulles d'eau et d'oxyde de carbone dans la masse vitreuse des particules.La teneur en eau y est faible,en moyenne de $0,5 \%$, mais inégalement répartie.

(1) Les fondants anhydre fondus sont disponibles chez ICPH SA, rue Jules Ferry 32, 54 Laxou (France).

(2) Le matériel en platine est disponible chez Engelhard-Clal, 45, rue de Paris, F-93130 Noisy-Le-Sec.

(3) Perl'X3 perleuse automatique de Philips. 